\title{
Verrechnung von Leistungen aus Kapitel 02.03 «Delegierte Psychotherapie in der Arztpraxis»
}

Voraussetzung für die Verrechnung von Leistungen aus TARMED-Kapitel 02.03 «Delegierte Psychotherapie in der Arztpraxis» ist nicht nur die erforderliche Dignität des delegierenden Arztes, sondern auch eine sogenannte Spartenanerkennung, nämlich für die Sparte «Delegierte Psychotherapie in der Arztpraxis». Dies ist nicht etwa eine Schikane, sondern notwendig, weil nach dem Willen der TARMED-Partner nicht nur die delegierenden Ärzte sondern auch die ausführenden nichtärztlichen Therapeutinnen und Therapeuten bestimmte Ausbildungskriterien erfüllen müssen.
Nach längerer Diskussion hat nun die dafür zuständige TARMED-PaKoDig der FMH grünes Licht gegeben, um die Anerkennungsverfahren für die Sparte «Delegierte Psychotherapie in der Arztpraxis» durchzuführen.

In Ausführung dieses Auftrags hat der FMHTarifdienst kürzlich an alle potentiellen Erbringer von Leistungen aus dem TARMED-Kapitel 02.03 den Selbstdeklarationsbogen für die Spartenanerkennung «Delegierte Psychotherapie in der Arztpraxis» mit folgendem Begleittext versandt:

Olten, 15. Februar 2006

\section{Delegierte Psychotherapie in der Arztpraxis}

Sehr geehrte/r Frau/Herr Doktor

Gemäss Vereinbarung über die Anerkennung von Sparten - Anhang zu den TARMED-Rahmenverträgen - unterliegt die Sparte «Delegierte Psychotherapie in der Arztpraxis» einer sogenannten Anerkennung, damit die Leistungen von TARMED-Kapitel 02.03 mit den Versicherern abgerechnet werden können.

Die FMH hat es im Auftrag der TARMED-Partner übernommen, das Anerkennungsverfahren durchzuführen. Es stellt, gleich wie TARMED-Anerkennungsverfahren in anderen Bereichen, auf eine Selbstdeklaration derjenigen Leistungserbringer $\mathrm{ab}$, welche die entsprechenden Leistungen erbringen und abrechnen wollen.

Mögliche Erbringer für TARMED-Leistungen aus Kapitel 02.03 sind Inhaber/innen der folgenden qualitativen Dignitäten:

- Kinder- und Jugendpsychiatrie und -psychotherapie;

- Psychiatrie und Psychotherapie;

- Fähigkeitsausweis «Delegierte Psychotherapie»

sowie Ärztinnen und Ärzte, welche die entsprechenden Leistungen im Zusammenhang mit der Dignitätserhebung als Besitzstand angegeben haben. Diese können den Fähigkeitsausweis ab sofort beantragen. Die entsprechende Kontaktadresse ist: Sekretariat SGDP, Quellenstrasse 31, 8005 Zürich, Tel. 04320529 55, E-Mail: ruthbuchholz@bluewin.ch, Internet: www.sgdp.ch.

Nach unseren Informationen gehören Sie zu einer der Gruppen möglicher Leistungserbringer für delegierte Psychotherapie. Aus diesem Grund senden wir Ihnen in der Beilage den Selbstdeklarationsbogen für die Anerkennung der oben erwähnten Sparte. In der Beilage G (Rückseite Selbstdeklarationsbogen) finden Sie zu Ihrer Information die massgebende Vereinbarung über die Anerkennung von Sparten nach TARMED. Dort sind die Kriterien und das Verfahren für die Anerkennung der Sparte «Delegierte Psychotherapie in der Arztpraxis» festgehalten. 
Wenn Sie in Ihrer Praxis Leistungen aus TARMED-Kapitel 02.03 «Delegierte Psychotherapie in der Arztpraxis» erbringen und Sie weiterhin mit den Versicherern ohne Probleme abrechnen möchten, bitten wir Sie, den beigefügten Selbstdeklarationsbogen auszufüllen, zu unterzeichnen und bis spätestens 30. März 2006 zurückzusenden an: FMH Tarifdienst, Gösgerstrasse 8, 4600 Olten.

Weil die Anzahl möglicher Leistungserbringer für delegierte Psychotherapie recht gross ist, werden wir keine «Mahnrunde» durchführen. Ohne Ihre Nachricht bis zum obenerwähnten Datum nehmen wir an, dass in Ihrer Praxis keine Leistungen aus TARMED-Kapitel 02.03 erbracht werden und wir Sie nicht auf die entsprechende Liste aufnehmen sollen.

Freundliche Grüsse
FMH
Karl Bachofen,
Dr. med. Olivier Kappeler, Projektleiter Tarifdienst Mitglied Zentralvorstand

\section{Das Wichtigste in Kürze}

- Das Fähigkeitsprogramm, das die Voraussetzungen für den Erwerb des Fähigkeitsausweises «Delegierte Psychotherapie» regelt, ist auf der Website des Sekretariates AWF zu finden (www.fmh.ch/awf $\rightarrow$ Weiterbildung $\rightarrow$ Weiterbildungs- und Fähigkeitsprogramme $\rightarrow$ Fähigkeitsprogramme $\rightarrow$ Delegierte Psychotherapie). Zuständig für die Erteilung des Ausweises ist das Sekretariat der Schweizerischen Gesellschaft für delegierte Psychotherapie (SGDP), Quellenstrasse 31, 8005 Zürich, Tel.04320529 55, E-Mail: ruthbuchholz@ bluewin.ch, Internet: www.sgdp.ch.

- Der Selbstdeklarationsbogen für die Anerkennung der Sparte «Delegierte Psychotherapie in der Arztpraxis» ist zu finden auf www. fmh.ch $\rightarrow$ Unsere Dienstleistungen $\rightarrow$ Tarife $\rightarrow$ Tarmed $\rightarrow$ Delegierte Psychotherapie; Selbstdeklarationsbogen für die Arztpraxis. Den ausgefüllten Fragebogen senden Sie bitte an den FMH Tarifdienst, Gösgerstrasse 8, 4600 Olten.

- Für Informationen im Zusammenhang mit der Spartenanerkennung «Delegierte Psychotherapie in der Arztpraxis» steht Ihnen der FMH Tarifdienst, Gösgerstrasse 8, 4600 Olten (Tel. 06228796 96, E-Mail: tarife@fmh.ch), gerne zur Verfügung. 